\title{
Cervical lymphadenopathy in childhood: nasopharyngeal carcinoma as a challenging diagnosis
}

\author{
Paula Martinez Viannaa, Cristiane Rúbia Ferreira ${ }^{b}$, \\ Pedro José dos Santos Neto ${ }^{c}$, Brenda Margatho Ramos Martines ${ }^{c}$
}

Vianna PM, Ferreira CR, Santos Neto PJ, Martines BMR. Cervical lymphadenopathy in childhood: nasopharyngeal carcinoma as a challenging diagnosis. Autopsy Case Rep [Internet]. 2012;2(4):53-60. http://dx.doi.org/10.4322/ acr.2012.033

\section{ABSTRACT}

Nasopharyngeal carcinoma (NPC) is a carcinoma that arises from the nasopharyngeal mucosa and differs from other head and neck carcinomas by its unique histologic, epidemiologic, and biologic characteristics. NPC is rare in most countries, especially Europe and North America. However, it has a high incidence in several regions of South China. The incidence variability of NPC, among different geographical and ethnic groups, indicates a combination of genetic susceptibility, infection by Epstein-Barr virus and environmental factors. NPC is classified into three histological subtypes according to the 1991 World Health Organization classification: squamous cell carcinoma, nonkeratinizing carcinoma, and basaloid squamous cell carcinoma. The symptoms of patients with NPC are related to the primary tumor site and the degree of dissemination. Therefore, patients can remain asymptomatic during a long period of time. Imaging exams and biopsy of the tumor mass generally are sufficient to establish the diagnosis. NPC is a rare disease among children. The authors report a case of a 12-year-old boy who sought medical attention complaining of a progressive growing tumoral mass on the right side of the neck. The computed tomography images of the head and neck and the histological examination of a cervical lymph node biopsy diagnosed a metastatic NPC.

Keywords: Nasopharyngeal carcinoma; Lymph node excision; Tomography, x-ray computed; Immunohistochemistry.

\section{CASE REPORT}

A 12-year-old male patient, previously healthy, sought medical attention complaining of a progressive growing tumoral mass on the right side of the neck, during the last month. The patient referred fever and oral bleeding since the beginning of the symptoms. He denied weight loss and night sweats. His primary care physician prescribed antibiotics, without any improvement.

On physical examination, three painless enlarged lymph nodes were present on the anterior and posterior, right cervical chain, and on the

\footnotetext{
a Department of Pathology - Faculdade de Medicina - Universidade de São Paulo, São Paulo/SP - Brazil.

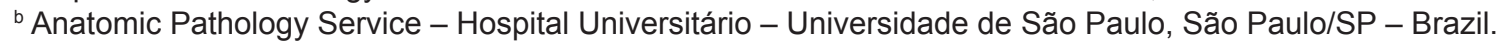

c Diagnostic Imaging Service - Hospital Universitário - Universidade de São Paulo, São Paulo/SP - Brazil.
}

Copyright $\odot 2012$ Autopsy and Case Reports - This is an Open Access article distributed of terms of the Creative Commons Attribution NonCommercial License (http://creativecommons.org/licenses/by/3.0/) which permits unrestricted non-commercial use, distribution, and reproduction in any médium provided article is properly cited. 
anterior left cervical chain, measuring up to 5.0 $\mathrm{cm}$. On palpation, all nodules were hard, fixed to surrounding tissues, without fluctuation points, and showed well-defined limits. The rest of the physical examination was unremarkable. Computed tomography (CT) of the neck revealed multiple, bilateral nodular masses images, some coalescent, on the pharyngeal, submandibular regions, and along the carotid arteries, measuring up to $4.0 \mathrm{~cm}$. Facial and abdominal CT were normal (Figures 1 and 2).

Laboratory workup was normal, and serology for toxoplasmosis (IgG and $\operatorname{lgM}$ ), cytomegalovirus, hepatitis $B$ virus, hepatitis $C$ virus, hepatitis A virus, HIV, and syphilis were all negative. Serology for Epstein-Barr virus (EBV) was IgG positive and $\lg M$ negative.

An incisional biopsy was performed, and multiple, irregular fragments were removed, measuring $2.0 \times 1.0 \times 0.4 \mathrm{~cm}$. All samples were submitted to histopathological evaluation, which revealed marked fibrosis and reactive lymphoid tissue with expanded paracortical zones. Focal areas of micro abscesses and cohesive groups of atypical cells with monotonous vesicular nuclei, prominent nucleoli, and lightly eosinophilic
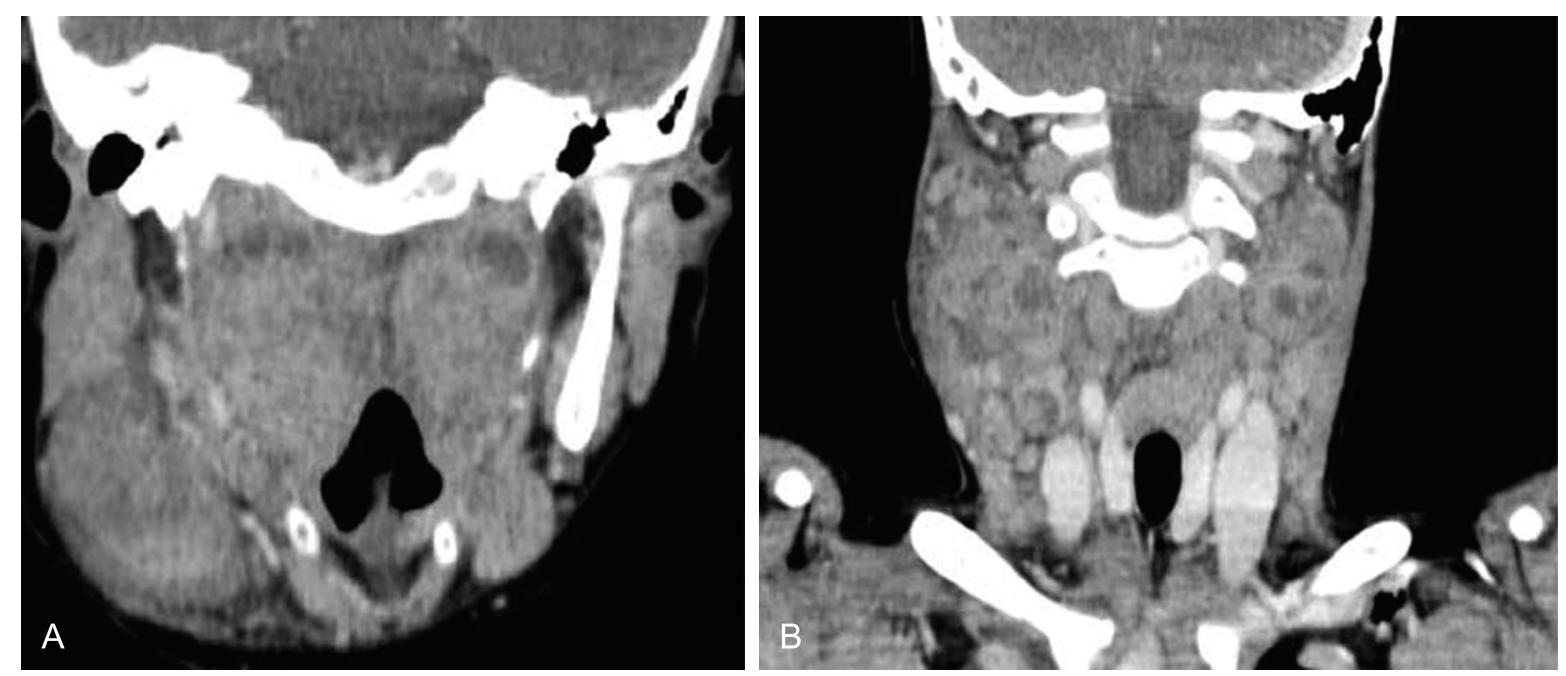

Figure 1 - Multidetector computed tomography of the head and neck. Coronal reformatted images after intravenous contrast injection. A - Infiltrating rhinopharyngeal masses spreading to the retro and parapharyngeal spaces; B - Multiple confluent lymph nodes with cystic/necrotic central areas scattered in various cervical plans, bilaterally.
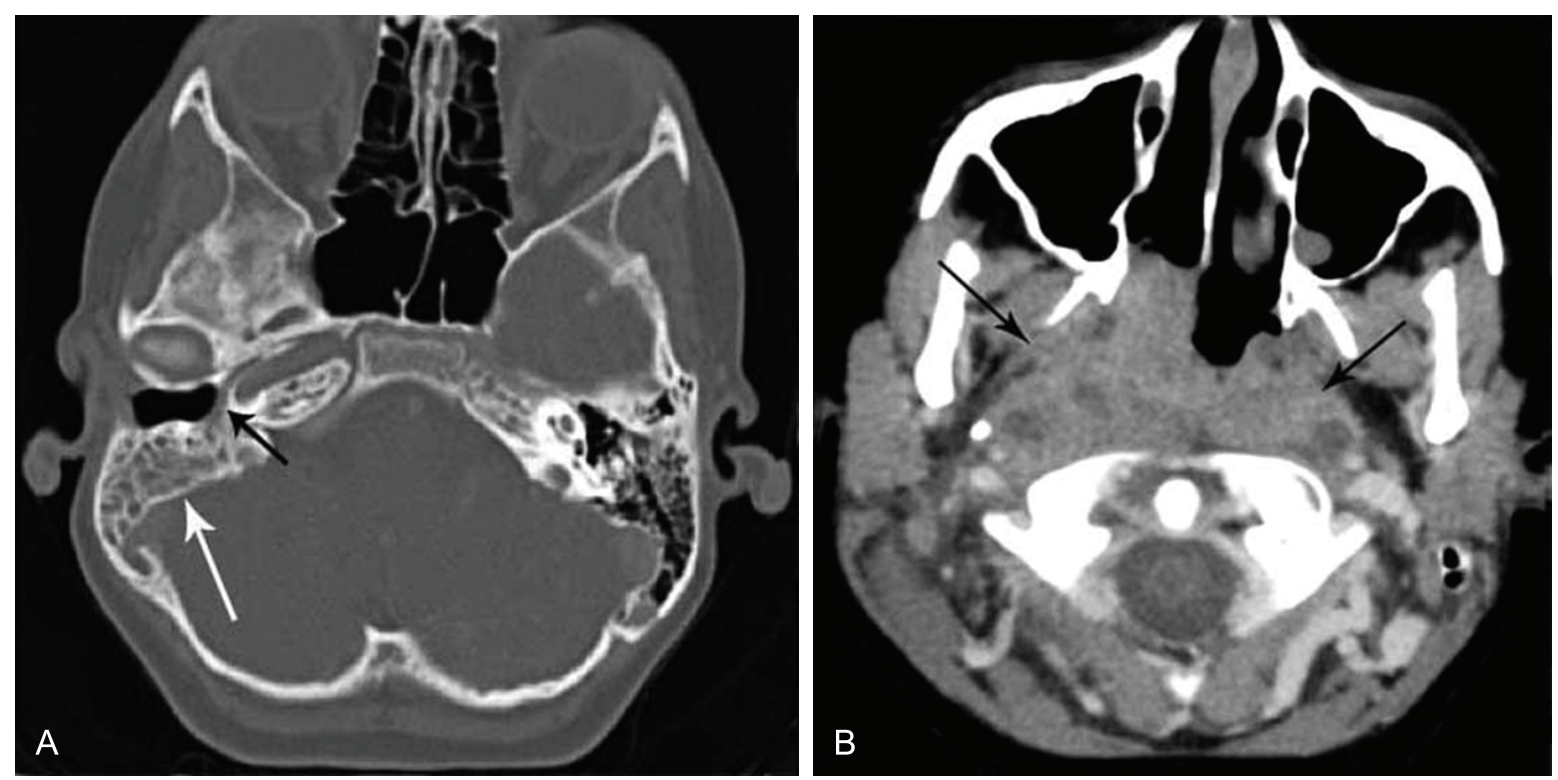

Figure 2-Axial computed tomography of the head and neck. A - (Bone window) hypodense material fulfilling the mastoid cells (white arrow) and the middle ear (black arrow), compatible with ostomastoiditis; B - After intravenous injection of the contrast, rhinopharyngeal infiltrative tumoral mass (black arrows), larger on the right side, obliterating Rosenmüller's fossa. 
cytoplasm with indistinct cell borders, resulting in a syncytial appearance were present (Figure 3). The immunohistochemical study is summarized in Table 1 and Figure 4.

The histological findings combined with the immunohistochemical results were consistent with metastatic lymphoepithelial carcinoma. Based on histological diagnosis, endoscopic exam of the nasopharyngeal cavity was undertaken, resulting in a disclosure of a suspicious mass localized on the nasopharynx, confirmed by CT images (Figure 5).
Given the histological, clinical, and tomographic findings, diagnosis was concluded as lymph node metastatic undifferentiated nonkeratinizing nasopharyngeal carcinoma (NPC).

\section{DISCUSSION}

We report a case of a 12-year-old boy with a cervical mass that revealed a metastatic NPC after histopathological examination. NPC is
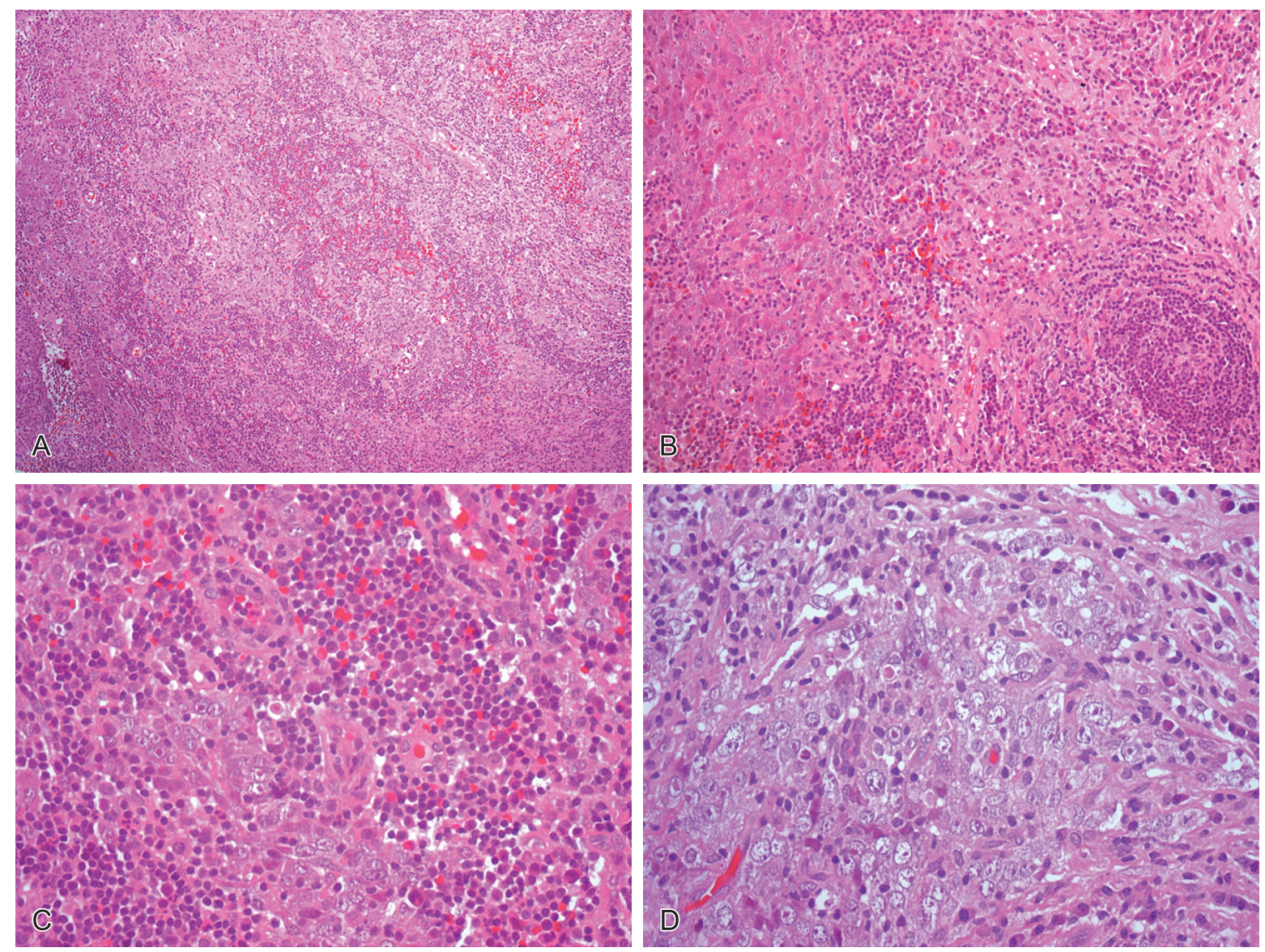

Figure 3 - A - Fibrosis and reactive lymphoid tissue; B - Islands of atypical cells with syncytial appearance; $\mathbf{C}$ and $\mathbf{D}$ - Atypical cells showing monotonous vesicular nuclei with prominent nucleoli, and lightly eosinophilic cytoplasm with indistinct cell borders (H\&E).

Table 1 - Immunohistochemical panel used for diagnosis

\begin{tabular}{ccccc}
\hline Antigen & Clone & Dilution & Manufacturer & Result \\
\hline AE1-AE3 & AE1-AE3 & $1: 2000$ & Cell Marque & Positive on atypical cells \\
EBV & CS1/CS2/CS3/CS4 & $1: 2000$ & DAKO & Positive on atypical cells \\
CD20 & L26 & $1: 6000$ & DAKO & Negative (positive on B lymphocytes) \\
CD3 & policlonal & $1: 1500$ & DAKO & Negative (positive on T lymphocytes) \\
CD68 & PG-M1 & $1: 5000$ & DAKO & Negative (positive on B histiocytes) \\
CD30 & Ber - H2 & $1: 400$ & DAKO & Negative (positive on focal reactive lymphocytes) \\
CD21 & $2 G 9$ & $1: 1200$ & Novocastra & Negative (positive on dendritic cells) \\
CD138 & MI15 & $1: 1200$ & DAKO & Negative (positive on plasmocytes \\
\hline
\end{tabular}



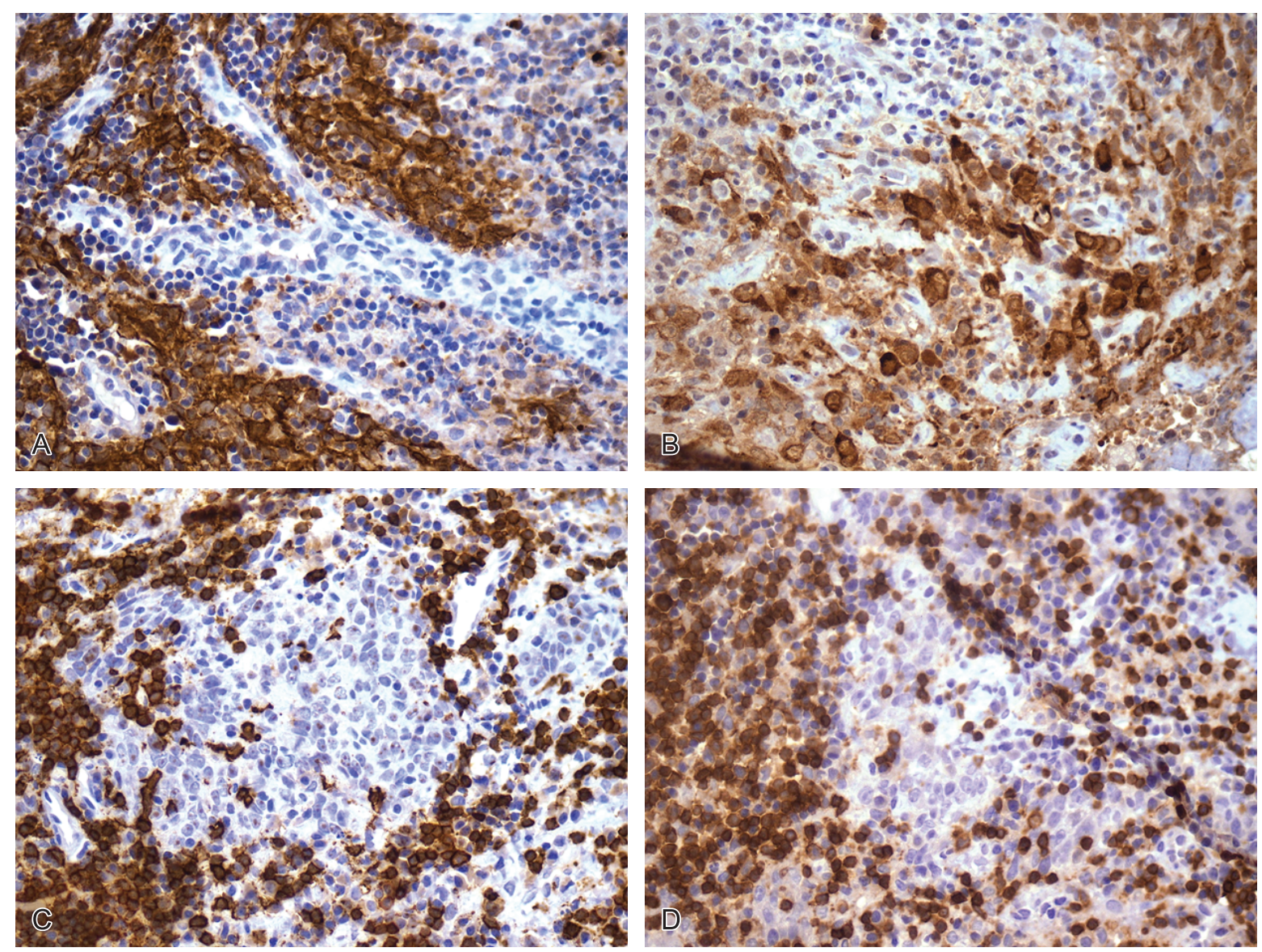

Figure 4-Immunohistochemistry. A - AE1-AE3 positive on atypical cells; B - EBV (LMP1) positive on atypical cells; C - CD20 positive only on small lymphocytes B; D - CD3- positive only on the small T lymphocytes.

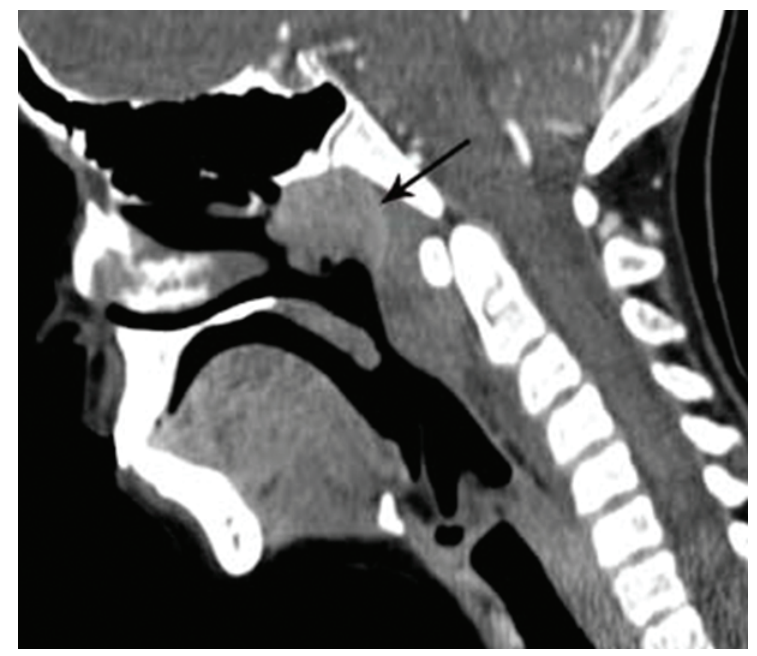

Figure 5 - Multidetector computed tomography of the head and neck. Sagittal reformatted images after intravenous contrast injection, showing an infiltrative mass in the rhinopharinx (arrow).

a carcinoma that arises from the nasopharyngeal mucosa and differs from other head and neck carcinomas by its unique histologic, epidemiologic, and biologic characteristics. ${ }^{1}$ It has as synonyms lymphoepithelioma, lymphoepithelioma-like carcinoma, and lymphoepithelial carcinoma.
NPC is rare in most countries, especially in Europe and North America. In the United States, it accounts for $0.25 \%$ of all malignancies. ${ }^{2}$ However, it has a high incidence in several areas of Southern China, $^{3}$ where the incidence ranges from $15 \%$ to $18 \%$ of all malignancies. It also accounts for $10-20 \%$ of childhood malignancies in Africa. ${ }^{2}$ Its incidence varies from less than 1 per 100,000 person-years in low-incidence regions to more than 20 per 100,000 person-years in endemic regions. ${ }^{4}$ In high-risk groups, NPC incidence raises after the age of 30 years and peaks at 40-60 years, declining afterwards. Gender predominance is observed with the relation between men and women of 2-3/1.2,5 In general, populations that migrate from high to low risk areas retain much of the elevated risk seen in their country of origin. ${ }^{6}$

NPC is a rare disease among children. ${ }^{7}$ Although great variability does exist concerning racial and geographical groups, this neoplasia constitutes from $1 \%$ to $5 \%$ of all cancers and from $20 \%$ to $50 \%$ of all primary nasopharyngeal malignant tumors in children. $^{8-10}$ The mean age of NPC development is 13 years, and incidence is highest among males (male to female ratio 1.8:1) and Black people. ${ }^{11,12}$ 
The incidence variability of NPC between different geographical and ethnic groups indicates a combination of genetic susceptibility, infection by Epstein-Barr virus (EBV) and environmental factors (consumption of Chinese nitrosamine-rich salted fish and other salted food) in the pathogenesis of the disease..$^{13}$ High antibody titers to EBV antigens $(\lg G$ and $\lg A)$ are useful diagnostic markers. ${ }^{2}$

A stepwise progression of histological features has been recently described. Patches of dysplasia are the earliest recognizable lesions, which are associated with allelic losses on the short arms of chromosomes 3 and 9, which result in inactivation of several tumor suppressor genes, particularly $p 14, p 15$, and $p 16$. These dysplastic areas are the origin of the tumor, but are probably insufficient to lead its further progression. At this stage, latent EBV infection becomes critical and leads to the development of severe dysplasia. Gains of genes on chromosome 12 and allelic loss on $11 q, 13 q$, and $16 q$ result in invasive carcinoma. Metastases are associated with mutation of $p 53$ and aberrant expression of cadherins. ${ }^{14,15}$

NPC is divided into three histological subtypes in accordance with the World Health Organization (WHO) classification of 1978, which are: squamous cell carcinoma (WHO type I), nonkeratinizing carcinoma (WHO type II), and undifferentiated carcinoma (WHO type III). ${ }^{16}$ In the 1991 WHO classification, the squamous cell carcinoma subtype (keratinizing squamous cell carcinoma) was maintained. The last two subtypes of 1978 WHO classification were grouped in a single category of "nonkeratinizing carcinoma", and were subdivided as "differentiated" or "undifferentiated". The current WHO classification maintains the terminology of the 1991 classification, with the addition of a new category: basaloid squamous cell carcinoma.

Comparing keratinizing squamous cell carcinoma with nonkeratinizing carcinoma, a trend for locally advanced tumor growth in the former $(76 \%$ vs $55 \%)^{17}$ as well as a lower incidence of lymph node metastasis (29\% vs $70 \%) .{ }^{18}$ Keratinizing squamous cell carcinoma shows immunoreactivity for pancytokeratin, high molecular-weight cytokeratin, and focally epithelial membrane antigen.

The nonkeratinizing carcinoma can be subdivided in undifferentiated and differentiated subtypes. Syncytial-appearing large tumor cells with indistinct cell borders, round to oval vesicular nuclei, and large central nucleoli characterize the undifferentiated subtype, which is more common. The lymph nodes can be involved extensively or subtly; the tumor takes the form of islands and strands, being intermingled with variable numbers of lymphocytes, plasma cells, and eosinophils. In cases of cervical lymph node metastases, the differential diagnoses are Hodgkin lymphoma, anaplastic lymphoma, and diffuse large B-cell lymphoma. The differentiated subtype differs from the undifferentiated subtype by showing cellular stratification often paved by a plexiform growth, reminiscent of transitional cell carcinoma of the bladder. ${ }^{19}$ The tumor cells show well-defined cell borders and sometimes-vague intercellular bridges; there also may be exceptionally occasional keratinized cells. ${ }^{20}$

Nonkeratinizing NPC is associated with EBV infection in virtually $100 \%$ of cases, irrespective of the ethnic background of the patient. By contrast, EBV latent membrane protein-1 (LMP1) is usually positive in only $30-40 \%$ of cases, and the immunostaining is often patchy and weak. The simplest and most reliable way to demonstrate EBV is in-situ hybridization for EBV-encoded early RNA. Practically all tumor cells show a strong reaction for pan-cytokeratin (AE1/AE3, MNF-116). ${ }^{20}$ The case reported here, showed an immunohistochemistry expression of AE1/AE3 and EBV on atypical cells, and a negative reaction for other lymphoid markers, leading to the diagnosis of NPC.

NPC often originates from the pharyngeal recess, the Rosenmüller's fossa. The primary tumor may present as a smooth bulging mucosa, a discrete nodule with or without ulceration, or an infiltrating mass. ${ }^{21}$ In general, this tumor spread via nasopharyngeal mucosa and submucosa along adjacent muscle and adipose planes and into bone foramina. As in the case presented here, most patients present a disseminated disease on the first consultation. ${ }^{21,22}$

The symptoms of patients with NPC are related to the primary tumor site and its degree of dissemination. Because of the anatomical peculiarities of the nasopharynx and the insidious growth of the tumor in this area, the nasopharynx has been considered a "blind spot", inaccessible to clinical evaluation. Therefore, patients can remain in a lengthy asymptomatic period. ${ }^{21,22}$ In children, the most common presenting symptom is a painless mass in the upper neck, which may be quite large at the first medical consultation. Local tumor effects 
include nasal obstruction, blood-tinged drainage, bleeding, and conductive hearing loss, or serous otitis caused by obstruction of the Eustachian tubes. The two latter events can also cause earache and tinnitus. Headaches, facial pain, and neck pain may result from obstructive phenomena or invasion. Nasopharynx is a relatively clinically silent area. Therefore, isolated lymph nodes metastasis to the neck, without clinical or radiological evidence of disease in the nasopharynx, may be observed. ${ }^{12}$ In the case reported here, a tumoral mass was evidenced in the nasopharynx only after diagnosis was defined on lymph node metastasis.

NPC has the highest preponderance for regional lymph node metastasis among squamous cell carcinoma of the head and neck. ${ }^{23}$ The most commonly involved cervical lymph node regions include lateral retropharyngeal nodes and level II nodes with an overall probability of $69.4 \%$ and $70.4 \%$, respectively. Certain cervical lymph node groups, including level I, level VI, parotid, and supraclavicular nodes have a very low risk for metastasis. ${ }^{24}$ Distant metastases include the bones $(67 \%)$, lungs $(20 \%)$, liver $(30 \%)$, bone marrow $(23 \%)$, and mediastinum. ${ }^{12}$

Sultan et al. ${ }^{1}$ compared the clinical features and outcomes of children, adolescents ( $<20$ years old) and adults with diagnosis of NPC. Based either on the AJCC-6 staging system or on tumor volume, they observed that children presented a more advanced disease at diagnosis. However, their outcome was significantly better compared to those observed in adults. A higher fraction of favorable WHO types (II and III) was observed in children, but it does not fully explain the better prognosis. In the multivariate analysis, age was shown to be an independent prognostic factor, where children and adolescents have half the risk of death compared to young adults.

Imaging exams and biopsy samples of the tumor generally reveal the diagnosis. CT remains the imaging exam of choice adopted by most institutions for the evaluation of tumors of the head and neck. Beyond the staging of advanced cases, it can reveal subtle details concerning the cranial base involvement with lytic or sclerotic lesions. Other findings furnished by CT are: displacement and obliteration of cervical adipose plans; asymmetry of the shape and density of muscle planes; changes in the density of bone surrounding structures; and indirect signs of perineural dissemination such as foramina enlargement and bone fissures. ${ }^{2,21}$ CT is still used for radiotherapy planning, and in some centers, is used with positron emission tomography (PET) using 18F-FDG. PET/CT has been shown to be of value in NPC staging, detecting distant metastasis. ${ }^{2}$ In the case presented here, there was no evidence of the perineural dissemination on CT images. In this regard, magnetic resonance imaging (MRI) has shown greater diagnostic accuracy. MRI is an accurate test for detecting subclinical cancers missed at endoscopy and endoscopy biopsies, and is also useful for ruling out this diagnosis. The protocol for NPC includes unenhanced T1weighted images to detect skull base involvement and fat planes in at least an axial and sagittal plane. A T2-weighted fast spin-echo sequence in an axial plane is used for early parapharyngeal tumor spread, paranasal sinus invasion, middle ear effusions, and detection of cervical lymph nodes. Axial and coronal contrast-enhanced T1-weighted images (with or without fat suppression) are used to detect tumor extent, including perineural spread and intracranial extension of the tumor. Whole body MRI for metastatic deposits of NPC is not currently recommended. ${ }^{2}$ Lymph node involvement is suspected when node size exceeds $10 \mathrm{~mm}$ and when there is a peripheral ring enhancement by contrast medium and a central hypodensity. ${ }^{25}$

The treatment in children follows guidelines established for adults. The main treatment strategy for all cases of locoregional NPC has been highdose radiotherapy to the nasopharynx and involved lymph nodes of the neck, and a moderate dose of radiation to uninvolved nodes and surrounding tissue. By contrast, surgery is generally not feasible due to potentially inadequate margins of resection. Chemotherapy depends on metastatic or recurrent disease activity. ${ }^{12}$

Due to the low incidence of NPC in children, misdiagnosis or delayed diagnosis is frequent. Therefore, the disease has to be included in the differential diagnosis of lymph node enlargement since isolated lymph nodes metastasis to the neck, without clinical or radiological evidence of disease in the nasopharynx, may be observed.

\section{REFERENCES}

1. Sultan I, Casanova M, A Ferrari, Rihani R, RodriguezGalindo C. Differential features of nasopharyngeal carcinoma in children and adults: a seer study. Pediatr Blood Cancer. 2010;55:279-84. PMid:20582982. http:// dx.doi.org/10.1002/pbc. 22521 
2. Razek AAKA, King A. MRI and CT of nasopharyngeal carcinoma. AJR Am J Roentgenol. 2012;198:11-8. PMid:22194474. http://dx.doi.org/10.2214/AJR.11.6954

3. Spano JP, Busson P, Atlan D, et al. Nasopharyngeal carcinomas: an update. Eur J Cancer. 2003;39:2121-35. http://dx.doi.org/10.1016/S0959-8049(03)00367-8

4. Sun LM, Li CI, Huang EY, Vaughan TL. Survival differences by race in nasopharyngeal carcinoma. Am J Epidemiol. 2007;165:271-8. PMid:17090616. http://dx.doi. org/10.1093/aje/kwk008

5. Ferlay J, Bray F, Pisani P, Parkin DM. Globocan 2000: cancer incidence, mortality and prevalence worldwide, version 1.0. Lyon: IARC; 2001.

6. Buell P. Race and place in the etiology of nasopharyngeal cancer: a study based on California death certificates. Int J Cancer. 1973;11:268-72. http://dx.doi.org/10.1002/ ijc. 2910110204

7. Ayan I, Kaytan E, Ayan N. Childhood nasopharyngeal carcinoma: from biology to treatment. Lancet Oncol. 2003;4:13-21. http://dx.doi.org/10.1016/S14702045(03)00956-2

8. Ingersol L, Woo SY, Donaldson S, et al. Nasopharyngeal carcinoma in the young: combined MD Anderson and Stanford experience. Int J Radiat Oncol Biol Phys. 1990;4:881-87. http://dx.doi.org/10.1016/0360-3016(90)90008-8

9. Pao WJ, Husto HO, Douglass EC, et al. Pediatric nasopharyngeal carcinoma. Long-term follow-up of 29 patients. Int J Radiat Oncol Biol Phys. 1989;17:299-305. http://dx.doi.org/10.1016/0360-3016(89)90443-4

10. Deutsch M, Mercado R, Parsons JA. Cancer of nasopharynx in children. Cancer. 1978;41:1128-33. http:// dx.doi.org/10.1002/1097-0142(197803)41:3<1128::AIDCNCR2820410348>3.0.CO;2-S

11. Plowman PN. Rare tumors. In: Pinkerton CR, Plowman PN, editors. Paediatric Oncology. 2nd ed. Cambridge: Chapman and Hall; 1997. p. 561-75.

12. Ayan I, Altun M. Nasopharyngeal carcinoma in children: retrospective review of 50 patients. Int J Radiat Oncol Biol Phys. 1996;35:485-92. http://dx.doi.org/10.1016/S03603016(96)80010-1

13. Chan AT, Teo PM, Johnson PJ. Nasopharyngeal carcinoma. Ann Oncol. 2002;13:1007-15. http://dx.doi.org/10.1093/ annonc/mdf179

14. Chan ASC, To KF, Lo WK, et al. High frequency of chromosome $3 p$ deletion in histologically normal nasopharyngeal epithelia from Southern Chinese. Cancer Res. 2000;60:5365-70. PMid:11034072.

15. Hui AB, Lo KW, Leung SF, et al. Detection of recurrent chromosomal and losses in primary nasopharyngeal carcinoma by comparative genomic hybridization. Int J Cancer. 1999:82:498-503. http://dx.doi.org/10.1002/ (SICl)1097-0215(19990812)82:4<498::AID-IJC5>3.0.CO;2-S

16. Shanmugaratnam K, Sobin LH. Histological typing of upper respiratory tract tumours. Geneva: WHO; 1978.

17. Reddy SP, Raslan WF, Gooneratne S, Kathuria S, Marks JE. Prognostic significance of keratinization in nasopharyngeal carcinoma. Am J Otolaryngol. 1995;16:103-8. http://dx.doi. org/10.1016/0196-0709(95)90040-3

18. Neel 3rd HB. Nasopharyngeal carcinoma. Clinical presentation, diagnosis, treatment, and prognosis. Otolaryngol Clin North Am. 1985;18:479-90. PMid:2995899.

19. Shanmugaratnam K. Histological typing of tumours of the upper respiratory tract and ear. 2nd ed. Berlin: SpringerVerlag; 1991. http://dx.doi.org/10.1007/978-3-642-84474-4

20. Chan JKC, Bray F, McCarron R, et al. Nasopharyngeal carcinoma. In: Barnes EL, Everson JW, Rechert P, Sidransky $D$, editors. Pathology and genetics of head and neck tumours. Lyon: World Health Organization of Tumours, IARC Press; 2005. p. 85-97.

21. Miura T, Hirabuki N, Nishiyama K. Computed tomographic findings of nasopharyngeal carcinoma with skull base and Intracranial involvement. Cancer. 1990;65:29-37. http:// dx.doi.org/10.1002/1097-0142(19900101)65:1<29::AIDCNCR2820650109>3.0.CO;2-2

22. Su CY, Lui CC. Perineural invasion of the trigeminal nerve in patients with nasopharyngeal carcinoma: imaging and clinical correlations. Cancer. 1996;78:2063-69. http://dx.doi. org/10.1002/(SICI)1097-0142(19961115)78:10<2063::AIDCNCR5>3.0.CO;2-P

23. Sham JS, Choy D, Wei WI. Nasopharyngeal carcinoma: orderly neck node spread. Int J Radiat Oncol Biol Phys. 1990,19:929-33. http://dx.doi.org/10.1016/03603016(90)90014-B

24. Ho FC, Tham IW, Earnest A, Lee KM, Lu JJ. Patterns of regional lymph node metastasis of nasopharyngeal carcinoma: a meta-analysis of clinical evidence. BMC Cancer. 2012;12:98. PMid:22433671 PMCid:3353248. http://dx.doi.org/10.1186/1471-2407-12-98

25. Sham JST, Cheung YK, Choy D, Chan FL, Leong L. Nasopharyngeal carcinoma: CT evaluation of patterns of tumor spread. Am J Neuroradiol.1991;12:265-70. PMid:1902025. 


\section{Conflict of interest: None}

Submitted on: $16^{\text {th }}$ September 2012

Accept on: $15^{\text {th }}$ November 2012

Correspondence: Departamento de Patologia

Faculdade de Medicina da Universidade de São Paulo

Av. Dr. Enéas Carvalho de Aguiar, 155 - 10 andar - Cerqueira Cesar - São Paulo/SP - Brazil

CEP: 055403-000 - Phone: +55 (11) 2261.7966

E-mail: viannapaula@hotmail.com 